\title{
CLUSTERED PILOT TONES FOR CARRIER FREQUENCY OFFSET COMPENSATION IN OFDM SYSTEMS
}

\author{
Wei Zhang ${ }^{\dagger}$, Xiang-Gen Xia ${ }^{\ddagger}$, and P. C. Ching ${ }^{\dagger}$ \\ †Department of Electronic Engineering, The Chinese University of Hong Kong \\ Shatin, N. T., Hong Kong. Email: \{wzhang, pcching\}@ee.cuhk.edu.hk \\ \#epartment of Electrical and Computer Engineering, University of Delaware \\ Newark, DE, 19716, USA. Email: xxia@ee.udel.edu
}

\begin{abstract}
A new pilot tone placing and pilot sequence design is proposed to compensate the carrier frequency offset (CFO) in OFDM systems. Unlike the conventional approach where isolated pilot tones are used, in this paper every two pilot tones are clustered as a group and these groups are equally spaced. The pilot sequence is carefully designed, of which the left hand sided pilot symbol in each cluster is antipodal with the right hand sided one. The performance in terms of the pilot channel interference ratio (CIR) can be significantly improved by the proposed pilot scheme. Theoretical analysis shows that the clustered pilot tones can give a substantially lower CFO variance than that of the isolated pilot tones. Simulation results are presented to verify our newly proposed theory.
\end{abstract}

\section{INTRODUCTION}

Inter-channel Interference (ICI) induced by the carrier frequency offset (CFO) will lead to the loss of orthogonality of the subcarriers in OFDM receiver and thus severely degrade the performance of reception in terms of bit error rate (BER) [1]. Generally speaking, there are two approaches of tackling this problem. The first one is the self-ICI cancellation method that transmits antipodal data symbols in pairs of subcarriers by taking into the consideration that there is relatively little change of ICI effect in adjacent subcarriers [2]. This method can reduce the ICI significantly but in the expense of one half of the bandwidth efficiency being lost. The other method utilizes a few of the isolated pilot tones (subcarriers) that are scattered over the entire spectrum to estimate the CFO and rectify the received signal from the feedback of the estimates [3,4]. It is obvious that $\mathrm{CFO}$ estimation is vital for subsequent $\mathrm{CFO}$ compensation. The ICI and other undesirable noise that affect the pilot tones will inevitably degrade the performance of the estimation.

In this paper, a clustered pilot tone pattern is proposed, which combines the merits of the above existing two methods. By placing the antipodal pilot symbols in each tone pair, the ICI and noise for the pilot tone can be greatly decreased and thus resulting in a higher pilot CIR. From the analysis of the variance of the CFO estimate, it is known that the variance is inversely proportional to the CIR. Consequently, it will lead to a better and robust estimate in terms of estimation variance and block length.

This paper is organized as follows. In Section 2, the ICI analysis of a conventional OFDM system is given. The typical isolated pilot tone for CFO estimation is described in Section 3. The clustered pilot tone method is proposed in Section 4. The variance of CFO estimate under noisy environment is derived in Section 5. Simulation results are shown and discussed in Section 6. Finally the conclusion is given in Section 7.

\section{OFDM ICI ANALYSIS}

In an OFDM receiver, the received signal is mixed with a local oscillator signal which is $\Delta f$ above the carrier frequency $f_{c}$. Ignoring

This work was partially supported by a research grant awarded by the Hong Kong Research Grant Council. the effects of noise, after removing the cyclic prefix and taking the $N$ point DFT of the signal, the demodulated symbol at the $m$ th subcarrier of the $i$ th OFDM symbol is given by [4]

$$
z_{m, i}=\exp \left(j\left(\theta_{0}+(i-1) \phi\right)\right) \sum_{l=0}^{N-1} c_{l-m} a_{l, i}
$$

where, the weighting coefficients, $c_{l-m}$, is given by

$$
\begin{aligned}
c_{l-m} & =\frac{1}{N} \sum_{k=0}^{N-1} \exp \left(\frac{j 2 \pi k(l-m+\Delta f T)}{N}\right) \\
& =\frac{1}{N} \frac{\sin (\pi(l-m+\Delta f T))}{\sin \left(\frac{\pi(l-m+\Delta f T)}{N}\right)} \\
& \times \exp \left(j \pi\left(\frac{N-1}{N}\right)(l-m+\Delta f T)\right)
\end{aligned}
$$

and $a_{l, i}$ denotes the QPSK symbol transmitted from the $l$ th subcarrier of the $i$ th OFDM symbol. $\theta_{0}$ is the phase rotation between the phase of the receiver local oscillator and the carrier phase at the begining of the received signal. $T$ is the effective duration of one OFDM symbol excluding the guard interval $G . \phi=2 \pi \varepsilon(1+G / N)$ is the phase rotation induced by the CFO and the normalized CFO $\varepsilon=\Delta f T=\Delta f / f_{\text {sub }} .|\varepsilon|<0.5$ and $f_{\text {sub }}$ denotes the subcarrier spacing.

It can be seen that the decoded complex value $z_{m, i}$ consists of a desired component due to $a_{m, i}$ as well as other terms due to the interferences. Here,

$$
z_{m, i}=\exp \left(j\left(\theta_{0}+(i-1) \phi\right)\right)\left(c_{0} a_{m, i}+u_{m, i}\right)
$$

where

$$
u_{m, i}=\sum_{l=0, l \neq m}^{N-1} c_{l-m} a_{l, i}
$$

is the ICI for the $m$ th subchannel and $c_{0}=\operatorname{sinc}(\Delta f T)$.

Fig.1 shows the complex weighting coefficients $c_{-N+1} \cdots c_{0} \cdots c_{N-1}$ for the case of $\Delta f T=0.1$ and $N=16$. It demonstrates that both real and imaginary parts of the coefficients are gradually changed with respect to the subcarrier index except several coefficients around the zero subcarrier offset.

Fig. 2 shows the magnitude of the adjacent weighting coefficients for different subcarrier offsets. The comparison illustrates that the first and second order difference of the coefficients can greatly reduce most of the interference power. This property has been used in [2] to cancel the ICI by transmitting antipodal data symbols at each pair of subcarriers while sacrificing one half of the bandwidth.

\section{TYPICAL PILOT TONES FOR CFO ESTIMATION}

One method of compensating the carrier frequency offset is to estimate the CFO from a few of isolated pilot tones embedding in the 


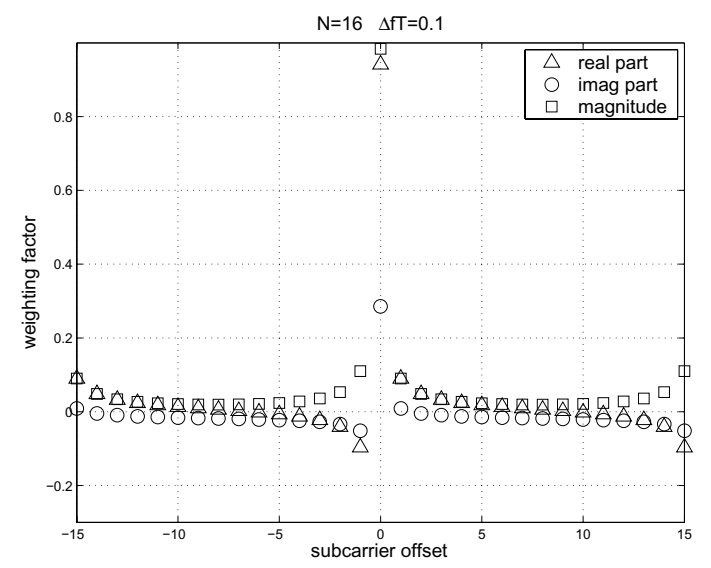

Figure 1: Real and imaginary components of the complex weighting coefficients

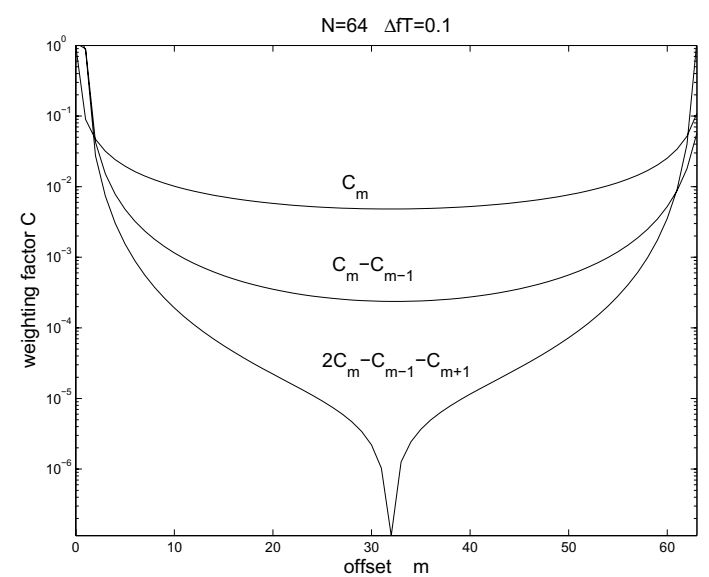

Figure 2: Magnitude of difference of adjacent coefficients vs. subcarrier offset for $\Delta f T=0.1$

received stream. Once the offset value is estimated, it will be sent back to the front end of the receiver to correct the received signal. From (3), it can be seen that each demodulated symbol is corrupted by ICI from all other subchannels.

Assume that the $m$ th subcarrier is one of the pilot tones which is scattered over the entire spectrum, the demodulated symbol of this tone in the $(i+1)$ th OFDM symbol is given by

$$
z_{m, i+1}=\exp \left(j\left(\theta_{0}+i \phi\right)\right)\left(c_{0} a_{m, i+1}+u_{m, i+1}\right) .
$$

Using only the $m$ th pilot tone, an unbiased estimate of the CFO is constructed from a block of $L$ symbols as [3, 5],

$$
\hat{\varepsilon}_{m}=\frac{1}{2 \pi(1+G / N)} \arctan \frac{\operatorname{Im} \sum_{i=1}^{L-1} z_{m, i}^{*} z_{m, i+1}}{\operatorname{Re} \sum_{i=1}^{L-1} z_{m, i}^{*} z_{m, i+1}}
$$

Taking account into all the pilot tones, $\bar{\varepsilon}=\frac{1}{P} \sum_{m=1}^{P} \hat{\varepsilon}_{m}$, where $P$ denotes the total number of pilot tones.

From (6), it is seen that the performance of the CFO estimate is dependent on the pulse pair $z_{m, i}^{*} z_{m, i+1}$. Consider a slow fading environment, the variance of the phase estimate from the pulse pairs is given by [6]

$$
\operatorname{var}(\phi)=\frac{1}{(L-1)^{2}}\left(\frac{1}{C I R}+\frac{L-1}{2} \frac{1}{C I R^{2}}\right)
$$

where CIR denotes the pilot channel interference ratio and has

$$
C I R=\frac{\left|c_{0}\right|^{2}}{\sum_{l=1}^{N-1}\left|c_{l}\right|^{2}}
$$

The variance of the CFO estimate shows that it is inversely proportional to the CIR of pilot tone. Thus it can be lowered by reducing the ICI on the pilot tone. To this end, the new pilot tone based $\mathrm{CFO}$ estimation method is provided in the following section.

\section{CLUSTERED PILOT TONES}

\subsection{Clustered Pilot Tone Design}

Unlike the conventional approach, in this paper, the pilot tones are organized into pairs/clusters. In each pair/cluster, the adjacent pilot symbols to be transmitted are always set to be antipodal. That is, $a$ is transmitted at the left pilot tone and $-a$ at the right pilot tone in each cluster. This results in cancellation of most of the ICI in the received pilot symbols.

Since $a_{m, i}=-a_{m+1, i}$ for $m \in S_{l}$, the decoded value for the left pilot tone at the $m$ th carrier is given by

$$
\begin{aligned}
z_{m, i} & =\exp \left(j\left(\theta_{0}+(i-1) \phi\right)\right)\left[\left(c_{0}-c_{1}\right) a_{m, i}\right. \\
& \left.+\sum_{l \in S_{l}, l \neq m}\left(c_{l-m}-c_{l-m+1}\right) a_{l, i}+\sum_{l \notin S} c_{l-m} a_{l, i}\right]
\end{aligned}
$$

where $S_{l}$ and $S$ denote the set of left pilot tones and of all pilot tones, respectively. Similarly, the decoded symbol at the right adjacent pilot tone is given by

$$
\begin{aligned}
z_{m+1, i} & =\exp \left(j\left(\theta_{0}+(i-1) \phi\right)\right)\left[\left(c_{-1}-c_{0}\right) a_{m, i}\right. \\
& \left.+\sum_{l \in S_{l}, l \neq m}\left(c_{l-m-1}-c_{l-m}\right) a_{l, i}+\sum_{l \notin S} c_{l-m-1} a_{l, i}\right]
\end{aligned}
$$

Next the values for the clustered pilot tones should be subtracted in pairs. It further reduces the ICI

$$
\begin{aligned}
\mathbf{Z}_{m, i} & =z_{m, i}-z_{m+1, i} \\
& =\exp \left(j\left(\theta_{0}+(i-1) \phi\right)\right)\left[\left(2 c_{0}-c_{1}-c_{-1}\right) a_{m, i}\right. \\
& +\sum_{l \in S_{l}, l \neq m}\left(2 c_{l-m}-c_{l-m+1}-c_{l-m-1}\right) a_{l, i} \\
& \left.+\sum_{l \notin S}\left(c_{l-m}-c_{l-m-1}\right) a_{l, i}\right]
\end{aligned}
$$

Substituting (9) into (6), an estimate for the normalized CFO can be obtained.

\subsection{Pilot Sequence Design}

The goal of pilot sequence design is to minimize the variance of the phase estimate. The phase variance of the clustered pilot tones with unknown pilot symbols is of the form

$$
\operatorname{var}(\phi)=\mathbf{E}\left[\frac{1}{(L-1)^{2}}\left(\frac{1}{C I R_{m}}+\frac{L-1}{2} \frac{1}{C I R_{m}^{2}}\right)\right]
$$

where $C I R_{m}$ denotes the $m$ th pilot channel interference ratio.

For the clustered pilot tones, the signal power for $\mathbf{Z}_{m, i}$ is

$$
\mathbf{E}\left(\left|\mathbf{C}_{m}\right|^{2}\right)=\left|2 c_{0}-c_{1}-c_{-1}\right|^{2}\left|a_{m, i}\right|^{2}
$$

Since the data symbol is a zero mean random process and independent to the pilot symbol, the ICI power for $\mathbf{Z}_{m, i}$ is given by

$$
\begin{aligned}
\mathbf{E}\left(\left|\mathbf{I}_{m}\right|^{2}\right) & =\left|\sum_{l \in S_{l}, l \neq 0}\left(2 c_{l}-c_{l+1}-c_{l-1}\right) a_{l, i}\right|^{2} \\
& +\sum_{l \notin S}\left|c_{l}-c_{l-1}\right|^{2}
\end{aligned}
$$




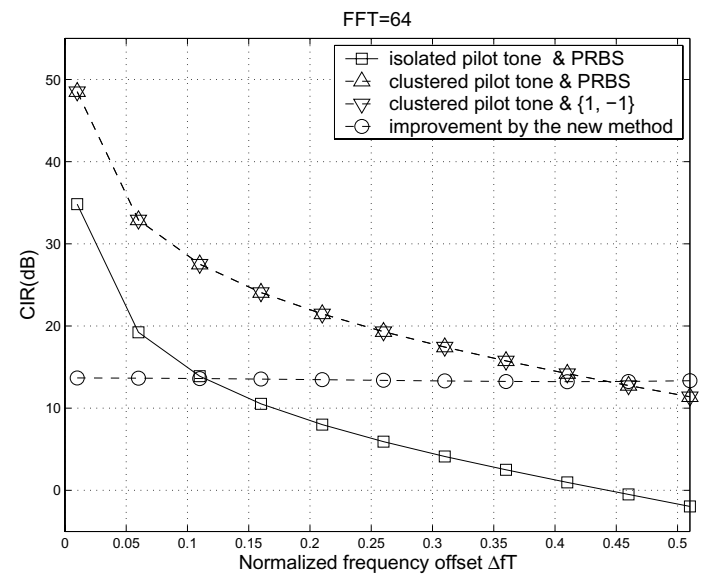

Figure 3: Pilot CIR versus frequency offset

It is shown in Fig. 2 that $\left|2 c_{l}-c_{l+1}-c_{l-1}\right|^{2} \ll\left|c_{l}-c_{l-1}\right|^{2}$ for any $l \in S_{l}$ and $l \neq 0$, so (12) can be further reduced to $\mathbf{E}\left(\left|\mathbf{I}_{m}\right|^{2}\right)=$ $\sum_{l \notin S}\left|c_{l}-c_{l-1}\right|^{2}$. Therefore, the CIR of the $m$ th clustered pilot tones yields

$$
C I R_{m}=\frac{\left|2 c_{0}-c_{1}-c_{-1}\right|^{2}\left|a_{m, i}\right|^{2}}{\sum_{l \notin S}\left|c_{l}-c_{l-1}\right|^{2}}
$$

Examining (10), the variance is a quadratic form of the inverse of the $C I R_{m}$ and can be approximated into linear form by discarding the second order term when $C I R_{m}$ is very large. After the approximation, the minimization of the variance is equivalently

$$
\min \left\{\mathbf{E}\left(\frac{1}{C I R_{m}}\right)\right\} \equiv \min \left\{\mathbf{E}\left(\frac{1}{\left|a_{m, i}\right|^{2}}\right)\right\}
$$

The suboptimum pilot sequence are obtained when (13) is satisfied and subject to the power constraint, $\sum_{m \in S_{l}}\left|a_{m, i}\right|^{2}=E_{p} / 2$, where $E_{p}$ is the total pilot power in one OFDM symbol.

It is found that such suboptimum sequence should be the sequence with equal magnitude for each symbol [7]. Assume that the total pilot power equals the total number of pilots, the $C I R$ for the clustered pilot tone with such unit magnitude sequence is equal to

$$
C I R_{c}=\frac{\left|2 c_{0}-c_{1}-c_{-1}\right|^{2}}{\sum_{l \notin S}\left|c_{l}-c_{l-1}\right|^{2}}
$$

One such suboptimal sequence is the pseudo random binary sequence (PRBS), the other one for the simple design of the OFDM system is the uniform sequence that transmits uniformly "1" at the left pilot tone and "- 1 " at the right one in each cluster.

Fig. 3 shows the CIR (in $\mathrm{dB}$ ) as a function of the normalized frequency offset, where $N=64$. The comparison validates the higher CIR of the clustered pilot tones due to the self-ICI cancellation than that of the isolated pilot tones for CFO estimation. It also demonstrates the close equivalence of the $C I R$ between using the random binary sequence and the uniform $\{1,-1\}$ for the clustered pilot tone methods. The improvement in CIR by the proposed method seems to be independent of CFO and shows a gain of about $13.7 \mathrm{~dB}$.

\section{VARIANCE OF CFO ESTIMATE IN AWGN}

When AWGN is considered, the demodulated symbols at the $m$ th pilot tone of the $i$ th OFDM symbol can be denoted by

$$
z_{m, i}=\exp \left(j\left(\theta_{0}+(i-1) \phi\right)\right)\left(c_{0} a_{m, i}+u_{m, i}+n_{m, i}\right)
$$

where $u_{m, i}$ is the ICI given by (4) and $n_{m, i}$ is the zero mean AWGN whose real part and imaginary part have the same variance of $\sigma_{n}^{2}$.

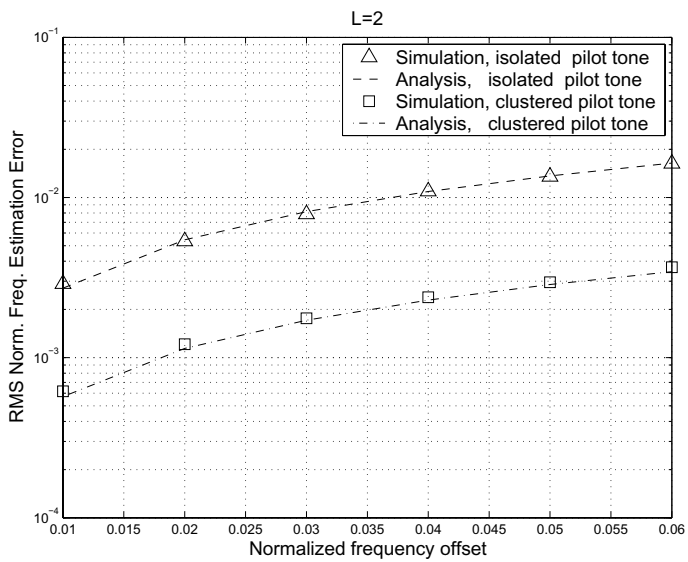

Figure 4: RMS of CFO estimate versus CFO $(L=2)$

Considering (7), the variance of the phase estimate can be similarly given by

$$
\operatorname{var}(\phi)=\frac{\lambda+\frac{L-1}{2} \lambda^{2}}{(L-1)^{2}}
$$

where $\lambda=\frac{1}{C I R}+\frac{1}{S N R}$. The $C I R$ is given by (8) and $S N R$ is defined as

$$
S N R=\frac{\left|c_{0}\right|^{2}}{2 \sigma_{n}^{2}}
$$

Similarly, for the case of clustered pilot tones the phase of variance is given by

$$
\operatorname{var}\left(\phi_{c}\right)=\frac{\lambda_{c}+\frac{L-1}{2} \lambda_{c}^{2}}{(L-1)^{2}}
$$

where $\lambda_{c}=\frac{1}{C I R_{c}}+\frac{1}{S N R_{c}}$ The $C I R_{c}$ is given by (14) and $S N R_{c}$ is defined as

$$
S N R_{c}=\frac{\left|2 c_{0}-c_{1}-c_{-1}\right|^{2}}{4 \sigma_{n}^{2}}
$$

Comparing (18) with (16), it will result in the approximation, $S N R_{c} \approx 2 \cdot S N R$. If SNR is high enough and CFO can be neglected, then it will give

$$
\operatorname{var}\left(\phi_{c}\right) \approx 0.5 \cdot \operatorname{var}\left(\phi_{S}\right)
$$

where the subscript $c$ and $s$ denotes the method of clustered pilot tone and isolated pilot tone used in the CFO estimation, respectively.

From Fig. 3, it is known that $C I R_{c} \approx 23.4 \cdot C I R_{s}$. If noise can be neglected and CFO is small, it will produce

$$
\operatorname{var}\left(\phi_{c}\right) \approx 0.04 \cdot \operatorname{var}\left(\phi_{S}\right)
$$

\section{SIMULATION RESULTS}

From (15) and Fig. 3, it can be seen that the accuracy of the CFO estimate is affected by three factors: the normalized CFO $\Delta f T$, the block length $L$ and the signal-to-noise ratio $S N R$. Next, simulations of CFO estimation on the three factors are performed, respectively, to illustrate the efficiency of the proposed method and compare with the square root of the variance.

\subsection{Effect of $\Delta f T$}

Simulation results of root mean square (RMS) error of CFO estimate are shown in Fig. 4 with respect to the frequency offset. It is clear that the RMS errors for both methods are increasingly proportional to the CFO. Moreover, the RMS error of the clustered pilot tone is always $1 / 5$ of the isolated pilot tone and thereby confirms the analysis in (20). 


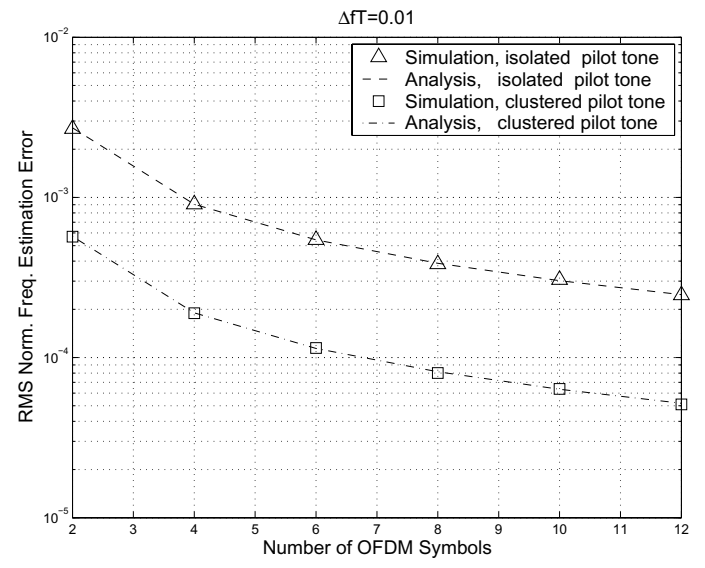

Figure 5: RMS of CFO estimate versus $L(\varepsilon=0.01)$

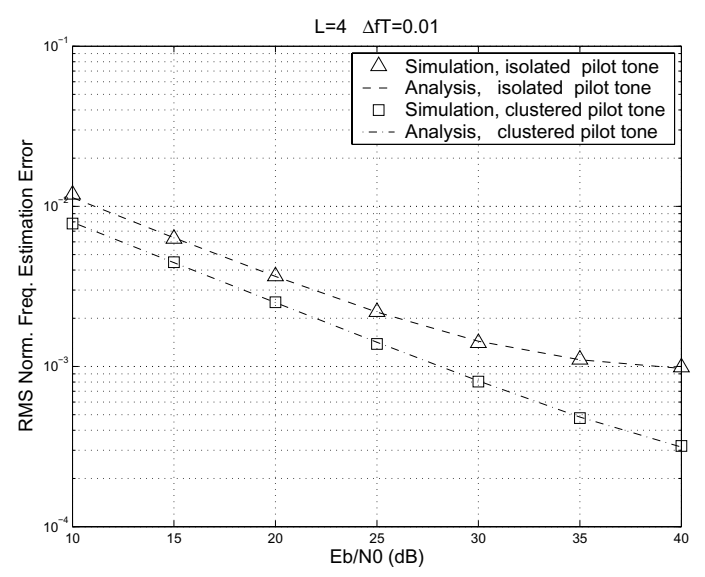

Figure 6: RMS of CFO estimate versus SNR $(L=4, \varepsilon=0.01)$

\subsection{Effect of $L$}

The effect of block length for the CFO estimation is depicted in Fig. 5.It is clear that the estimation variance decreases with an increasing block length and the clustered pilot tone always gives a smaller variance than the isolated pilot tone irrespectively to the block length. For example, when $L=2$ the variance from the clustered pilot tone is only $4 \%$ of that from the isolated pilot tone. It will be extremely useful for fast CFO estimation which is so required in real applications.

\subsection{Effect of $S N R$}

The comparison of RMS error of the estimate between both methods are illustrated with respect to the SNR for the normalized frequency offset of 0.01 and 0, in Fig. 6 and Fig. 7, respectively. The error floor of the CFO estimate in the case of isolated pilot tone shown in Fig. 6 tends to be higher than that in the clustered pilot tone. Fig. 7 confirms the conclusion of (19) in which the clustered pilot tone method can reduce the $3 \mathrm{~dB}$ noise as compared with the isolated pilot tone method.

\section{CONCLUSION}

A new pilot tone based CFO estimation method is proposed by transmitting the antipodal pilot symbols at the clustered pilot tones. Thus the ICI at pilot tone can be cancelled greatly and lead to a higher CIR. Through the analysis of the CFO variance, the higher CIR consequently results in a lower variance. Simulation results demonstrate the efficacy of the proposed method which gives a ro-

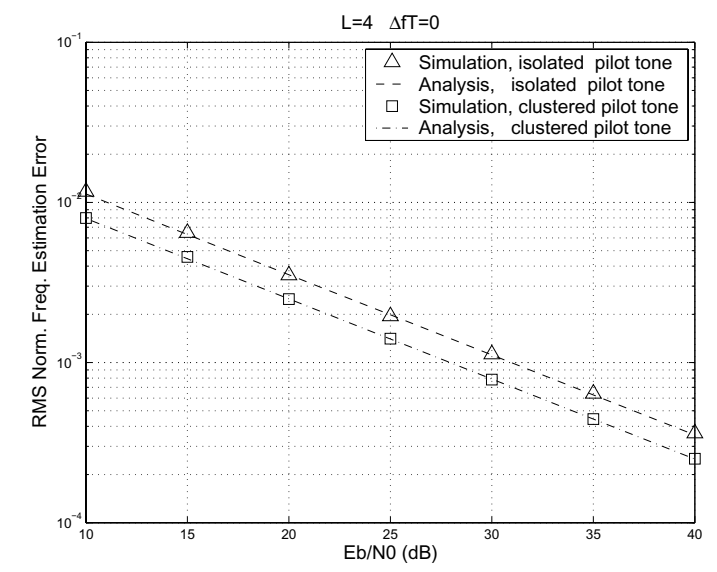

Figure 7: RMS of CFO estimate versus $\operatorname{SNR}(L=4, \varepsilon=0)$

bust estimate even under noise environments.

\section{REFERENCES}

[1] T. Pollet, M. Van Bladel, and Moeneclaey, "BER sensitivity of OFDM systems to carrier frequency offset and Wiener phase noise," IEEE Trans. Commun.,vol. 43, pp. 191-193, July 1995.

[2] Y. Zhao and S.-G. Häggman, "Intercarrier interference selfcancellation scheme for OFDM mobile communication systems ," IEEE Trans. Commun.,vol. 49, pp. 1185-1191, July 2001.

[3] J. van de Beek, M. Sandell, and P. O. Borjesson, "ML estimation of time and frequency offset in OFDM systems ," IEEE Trans. Signal Processing,vol. 45, pp. 1800-1805, July 1997.

[4] J. Armstrong, "Analysis of new and existing methods of reducing intercarrier interference due to carreier frequency offset in OFDM," IEEE Trans. Commun.,vol. 47, pp. 365-369, Mar. 1999.

[5] S. Kapoor, D. J. Marchok, and Y.-F. Huang, "Pilot assisted synchronization for wireless OFDM systems over fast time varying fading channels," in Proc. VTC'98, Ottawa, Canada, May 1998, pp. 2077-2080.

[6] Z. Wang and S. S. Abeysekera, "Frequency estimation using the pulse-pair method in different fading enviroments," in Proc. ICASSP'03, Hong Kong, 6-10 April 2003, vol.4, pp. 517-520.

[7] C. Tellambura, M. G. Parker, Y. J. Guo, S. J. Shepherd, and S. K. Barton, "Optimal sequences for channel estimation using discrete Fourier transform techniques," IEEE Trans. Commun.,vol. 47, pp. 230-238, Feb. 1999. 